\title{
Foundations and Applications of Intelligent Knowledge Exchange
}

\author{
S.J. Overbeek ${ }^{1}$, P. van Bommel ${ }^{2}$, H.A. (Erik) Proper $^{2}$ and D.B.B. Rijsenbrij ${ }^{2}$ \\ ${ }^{1}$ e-office B.V. \\ Dolderseweg 2, 3712 BP Huis ter Heide (Utrecht), The Netherlands, EU \\ Sietse.Overbeek@e-office.com \\ ${ }^{2}$ Institute for Computing and Information Sciences \\ Radboud University Nijmegen, Toernooiveld 1, 6525 ED Nijmegen, The Netherlands, EU \\ \{P.vanBommel, E.Proper, D.Rijsenbrij\}@cs.ru.nl
}

\begin{abstract}
Exchange of knowledge is becoming increasingly important to modern organizations. In this chapter is explained what this elementary knowledge exchange consists of and how a virtual workplace can support knowledge exchange between workers. A scenario from the medical domain illustrates how physicians can improve their knowledge exchange by utilizing the virtual workplace models introduced. Better adaptation to the rapidly changing nature of providing health care is a desirable effect of improved knowledge exchange between physicians. Explicit models concerning possible physical, social and digital contexts of knowledge exchange are discussed, as well as models which depict how knowledge relatedness enable intelligent knowledge exchange. Researchers studying virtual workplace models for industry and academic purposes belong to the intended audience of this chapter. Administrators of public sector or other non-profit agencies who wish to incorporate virtual workplace models and methods into their daily operations can also benefit from the contents discussed.
\end{abstract}

\section{Introduction}

The importance of knowledge and in particular the dissemination of knowledge is becoming increasingly important for organizations. An example can be found in the medical domain, clearly illustrated by Frank (2005) in that today's physicians continue to witness significant change in the nature of health care delivery. Practice is changing daily, with literally thousands of medical journals documenting the evolving understanding of biological, social and clinical sciences. Patients are treated in more 
diversified settings and spend less time in hospitals. In this environment a physician requires to acquire more knowledge than ever before so that the needs of their patients can be met.

Both academia and industry gradually anticipate on the aforementioned social developments by concentrating on the development of virtual workplaces so that knowledge dissemination improves. Studies which concentrate on the development of online work have been conducted by e.g. Malhotra and Majchrzak (2005) and Schaffers, Brodt, Pallot and Prinz (2006).

In this chapter, the general focus is on providing support by means of a virtual workplace so that knowledge exchange between workers improves. Before building such a virtual workplace, in-depth understanding of the support which a virtual workplace can deliver to improve knowledge exchange is necessary, together with comprehension of the reasons of that support. For this matter it implies a clear understanding of knowledge exchange and an elaboration of current computer-based support to improve knowledge exchange.

To better understand knowledge exchange and how a virtual workplace can support that, several models explaining possible contexts of knowledge exchange are depicted. Furthermore, specific support situations are distinguished in which a worker requests assistance from the virtual workplace when exchanging knowledge. This chapter will make clear how a virtual workplace is able to facilitate knowledge sharing using contextual models and support mechanisms. Section 2 discusses the basics of knowledge exchange and examples of computer-based support for knowledge exchange are introduced. Section 3 describes knowledge exchange from a physical, social and digital context. In section 4 a fundamental model of knowledge exchange is elaborated, followed by section 5 in which more sophisticated models are shown for improving knowledge exchange. Section 6 discusses future research topics and the chapter is concluded by section 7.

\section{Understanding Intelligent Knowledge Exchange}

To be able to better determine what kind of computer-based support is desired and feasible to improve knowledge exchange between workers, a better understanding of knowledge, knowledge exchange, and already available computer-based support for knowledge exchange is called for. Knowledge exchange occurs during organizational knowledge transformation processes and is part of organizational knowledge lifecycles. Both concepts will be discussed in this section to explore various different perspectives on knowledge transformation processes and knowledge lifecycles (each possibly taking a 
specific understanding of what knowledge is as a starting point). We will also stipulate our essential view on knowledge exchange and discuss how this view materializes in each of the discussed transformation processes and lifecycles.

\subsection{Definitions of Knowledge}

In the literature, many different definitions of knowledge pass in review. Dependent of which interpretation one chooses, our knowledge exchange definition in section 2.2 can be specialized using a specific definition of knowledge. Some of the definitions found in the literature are discussed in this section to better understand the notion of knowledge. In many definitions a distinction is made between tacit (or nowadays more often denoted as implicit) knowledge and explicit knowledge. There are also definitions which specifically focus on the tacit / implicit part or the explicit part.

Polanyi (1966) is recognized as the one who introduced the term tacit knowledge as a specific form of knowledge. He defined tacit knowledge as complex abstract knowledge that is totally individual, hard to formalize and to communicate, and introspective in nature. However, communication is a necessary prerequisite in order to exchange knowledge and therefore tacit knowledge as defined by Polanyi is also difficult to exchange with another worker. The 'knowledge resource' is often the human brain. So in order to exchange a tacit knowledge item, it must first be distilled from the brain and formulated in a way so that it is suitable for exchange. Dienes and Perner (1999) state that implicit knowledge comprises aspects of knowledge that are not differentiated or articulated. "For example the sentence 'The king is bald' ... presupposes or implicitly represents that there exists a king" (Pinku \& Tzelgov, 2005, p. 2). Perhaps the essence of what is meant in literature by tacit and implicit knowledge is concealed in the sentence: 'knowing without telling'.

Explicit knowledge is different in nature than tacit or implicit knowledge. According to Dienes and Perner (1999) explicit knowledge is knowledge which is represented by means of an internal state whose function is to indicate the knowledge. For example the sentence 'The rabbit is brown' explicitly denotes that the rabbit is brown. Nonaka and Takeuchi (1995) also consider tacit and explicit knowledge as two dimensions of knowledge in that tacit knowledge can be characterized by subjectivity, direct personal experience, qualitative nature, simultaneous processing and a practical base. Their definition of explicit knowledge comprises terms as objectivity, rationality, sequential processing and 'quantitative in nature'. Due to its characteristics explicit knowledge is easier to communicate and hence easier to exchange. 
There are obviously many more definitions to discuss in this section, but those already mentioned represent the more fundamental interpretations of knowledge which can be found in the literature. Other definitions can be found in e.g. Barwise (1989) and Siemieniuch and Sinclair (1999).

\subsection{A Definition of Knowledge Exchange}

There is literature discussing the topic of 'knowledge exchange' on itself and also specific ideas to provide computer-based support for knowledge exchange. Kuznets (1962) mentions that knowledge exchange flourishes in dense intellectual settings, and the more intellectual contact flourishes, the more knowledge is added to resources of knowledge. The research of Kuznets focuses on intellectual capital in large cities, while the research discussed in this chapter focuses specifically on exchange of knowledge between workers in an organizational setting.

Heterogeneity (in terms of different types of knowledge) is considered as an important factor in successfully exchanging knowledge (Berliant, Reed \& Wang, 2006). Less knowledge exchange occurs when individuals' types of knowledge are too diverse and when individuals' types are too similar. To determine the efficacy of knowledge exchange, a function is introduced in the research of Berliant, Reed and Wang to measure the ideal 'knowledge distance' between two individuals. Furthermore, their research specifically focuses on the relationships between knowledge exchange and population agglomeration. Cowan, Jonard and Özman (2004) associated knowledge exchange with the arousal of innovation in a community of actors, based on the idea that innovation is largely a result of knowledge exchange among a small group of agents. Cowan, Jonard and Özman specifically took the tacitness of knowledge into account in assessing innovative potential, therefore they indirectly adopted the definition of Polanyi (1966). Our definition is more generic with respect to the concept of knowledge.

In order to define a general view on knowledge exchange, which includes 'software agents' as part of the virtual workplace, we propose that knowledge flows from:

1. A worker to another worker.

2. A software agent to another software agent.

3. A software agent to another worker.

4. A worker to another software agent. 
The term 'software agent' is further explained in section 2.3. It is assumed that knowledge $K$ can be retrieved from a knowledge resource $K R$ and that knowledge can flow by means of a communication device and a communication medium. A knowledge resource is an entity from which knowledge can be subtracted, e.g. a human brain or any suitable hardware device. A communication device is an entity which is necessary to initiate a knowledge exchange event and eventually knowledge can flow by means of a communication medium. The relevant knowledge resources for a knowledge item can be depicted as $R: K \rightarrow \wp(K R)$, so that $R(x)=\left\{y_{1}, y_{2}\right\}$ is interpreted as: Knowledge item $x$ is retrieved from knowledge resource $y_{1}$ as well as from knowledge resource $y_{2}$.

Furthermore, it is assumed that a certain worker has a need for knowledge to benefit from a knowledge exchange event. That need for knowledge is influenced by what the worker already has retrieved from another knowledge resource. Weide and Bommel (2006) have already introduced the following function to measure one's need for knowledge:

$$
N: \wp(K) \times K \mapsto[0,1]
$$

$N(S, x)$ is interpreted as the residual need for knowledge item $x$ after the set $S$ has been presented to the worker, where $S \subseteq K$. So knowledge exchange involves the broadcasting of knowledge items between workers, or between a worker and a software agent and vice versa, with as specific goal to reduce the need for knowledge of a worker. No more knowledge exchange is necessary if $N(S, x)=0$. This definition of knowledge exchange is constructed in such a way that at least every one of the definitions of knowledge as mentioned in section 2.1 can be used for the notion of 'knowledge'. The knowledge input and output that a worker consumes respectively generates in the process of knowledge exchange can be depicted as $i, o: T \rightarrow(W \rightarrow \wp(K))$, where $T$ is the set of worker states (which differ from each other over time) and $W$ is the set of workers. A worker state is necessary to keep track of what a worker already has produced in terms of knowledge items and what a worker already has received in terms of knowledge items. The function $i\left(t_{1}, w_{1}\right)$ for instance determines the input in terms of knowledge items at state one of worker $w_{1}$. However, the state aspect will not be relevant for the more basic models of knowledge exchange until support relatedness is introduced in section 5.2. For notation simplicity knowledge input is indicated by the function $i_{1}\left(w_{1}\right)$ if the worker state is relevant (indicating state one of worker $\left.w_{1}\right)$ and the notation $i\left(w_{1}\right)$ is used if worker states are not relevant. The character $i$ is replaced by the character $o$ if knowledge 
output is concerned. Possible contexts of knowledge exchange are discussed in section 3 . A fundamental model of knowledge exchange is elaborated in section 4 followed by more sophisticated models in section 5 .

\subsection{The Term 'Software Agent'}

The term 'software agent' has been postulated in our view on knowledge exchange. According to Wooldridge and Jennings (1995), a software agent is an encapsulated computer system that is situated in some environment and that is capable of flexible, autonomous action in that environment. As can be distilled from this definition, software agents are autonomous, which means that they can function on their own, without requiring human support. It has the control over its own actions and internal state and it can decide whether or not to perform a requested action.

Software agents are designed to fulfill a specific purpose and have particular goals to achieve, exhibiting flexible and pro-active behaviour. Software agents are also often capable of 'social' behavior because they can communicate and cooperate with each other. Eventually, for software agents to be highly intelligent, it is desirable that they are able to learn as they react and interact with their external environment. In this case, a software agent should be able to exchange knowledge with the worker if that is the worker's wish and it should understand the specific need for knowledge which a worker has. A collection of software agents which improve knowledge exchange are part of the virtual workplace we focus on and can assist in improving knowledge exchange between workers. Software agents are further discussed in sections 3.4, 4.2 and 5.2.

\subsection{Knowledge Transformation Processes \& Knowledge Lifecycles}

Knowledge exchange is part of organizational knowledge transformation processes and organizational knowledge lifecycles. There are many different perspectives on knowledge transformation processes and lifecycles, each taking a specific understanding of what knowledge is as a starting point. This section takes up on the materialization of our essential view on knowledge exchange in each of the discussed knowledge transformation processes and lifecycles.

The research of Siemieniuch and Sinclair (1999) includes such a knowledge lifecycle in which our view on knowledge exchange can be materialized. According to Siemieniuch and Sinclair knowledge is not uniform and it has a lifecycle in a competitive environment. "In other words, if a company is to remain competitive, it must address the issues of new knowledge generation, its propagation across the organization, and its subsequent retirement" (Siemieniuch \& Sinclair, 1999, p. 1). A worker's specific need for knowledge as mentioned in section 2.2 can cause new knowledge generation to meet that 
worker's demands. Knowledge input and output is necessary to propagate knowledge across the organization. If the need for a knowledge item $x$ has reached zero and if the need for that knowledge item remains zero long enough, then the knowledge item will eventually deteriorate. Siemieniuch and Sinclair discuss that knowledge will age as the context changes, and humans will be intrinsic components in all processes involving the creation, utilization and retirement of knowledge.

The knowledge conversion model of Nonaka and Takeuchi (1995) is one of the most well-known models describing knowledge transformation processes within organizations. A knowledge transformation process involves all events which transform a certain knowledge type into another knowledge type, e.g. the events to transform implicit knowledge to explicit knowledge which is the case in the model of Nonaka and Takeuchi. Generation and consumption of knowledge as discussed in section 2.2 is required to distil the knowledge exchange situations that cause a conversion from implicit knowledge to explicit knowledge or vice versa, which are all classified in the model of Nonaka and Takeuchi. To illustrate one of those conversions, assume that a worker pair $w_{1}, w_{2}$ exchange knowledge and that the output of worker $w_{1}$ contains explicit knowledge and the input of worker $w_{2}$ contains implicit knowledge. This specific transformation from explicit to implicit is classified as 'socialization' by Nonaka and Takeuchi, because experiences are shared and implicit knowledge is created from explicit knowledge (such as shared mental models and technical skills).

Nonaka and Takeuchi (1995) focus on implicit and explicit knowledge when knowledge transformation processes are concerned. Strambach (2001), however, focuses on knowledge transformation processes between organizations by means of knowledgeintensive business services so that new knowledge is acquired by interactions between organizations. An organization on itself can codify or recombine newly gained knowledge and subsequently that recombined knowledge can be disseminated again among client firms causing the birth of new knowledge within the present client firms. In Strambach's model, knowledge exchange takes place on an organizational level, so when a certain organization $x$ has a need for knowledge it can gain new knowledge by exchanging knowledge with client firms. After this inter-organizational exchange process, organization $x$ then exchanges knowledge internally so that the acquired knowledge is codified or recombined. When applying our view on knowledge exchange on Strambach's model, the function $N(S, x)$ expresses the residual need of an organization for knowledge item $x$ after the set $S$ has been presented to the organization 
due to previous interactions with other organizations. In this case the set $S$ represents the knowledge profile of an organization as a whole.

We have chosen to discuss some examples to illustrate how our view on knowledge exchange materializes in certain knowledge transformation processes and lifecycles. Therefore the list of models discussed in this section is obviously not a complete overview of all existing models. However, other models describing knowledge transformation processes or knowledge lifecycles can be found in e.g. Holsapple and Singh (2001) and Koulopoulos, Spinello and Toms (1997).

\subsection{Examples of Current Computer-Based Support for Knowledge Exchange in the Virtual Workplace}

Internet, of course, has allowed the spread of knowledge without frontiers, but intelligent (web-based) software agents as mentioned in section 2.2 are also utilized when supporting knowledge exchange from a virtual workplace perspective. The research carried out by Li, Montazemi and Yuan (2006) shows an example of how software agents may assist users in the process of searching for acquaintances on the internet for exchanging musical knowledge. They have developed a web-based system which allowed users to perform four major tasks: entering music attribute preferences; selecting favourite music at a music site and creating a music collection; communicating with other subjects and manually find buddies; and evaluating the quality of manual- and agentfound buddies. This test system consisted of three major components: a music browser, a message board, and an agent-based buddy-finding system. The agent-based buddyfinding system decreased the burden of searching for the right acquaintances in order to exchange relevant and useful musical knowledge. Time which would be lost in a manual search process for acquaintances can now be used for other purposes and exchange of musical knowledge can be optimized due to the automatically discovered acquaintances. The focus area 'musical knowledge' does not relate with our research on improving knowledge exchange within the virtual workplace, but the proposed use of agent technology certainly does.

Groth (2004) has proposed a technological framework for supporting knowledge exchange in organizations. The framework depicts that communication (a prerequisite to exchange knowledge), awareness (of others' activities and availability), and information management (how to structure and reuse already existing information) are important aspects to consider when providing computer-based support for knowledge exchange.

Several software applications make use of the technological framework as proposed by Groth (2004) already, however these applications are not based on agent 
technology. One of those applications is called 'Mobile Elvin', which involves communication that is mainly synchronous (between desktop and mobile platforms) and from one person to a group of persons. With Mobile Elvin it is possible to not only pose a question to only one person, but also to a specific group of people within the organization. Depending of the communication device, the worker receives the message on a mobile device or a desktop. Asynchronous communication is supported by means of e-mail applications.

Ordinary discussion forums and news groups are two successful examples of software applications used for knowledge exchange to improve the quality of learning in organizations. However, these relatively simple mechanisms of cooperation present two main problems (López, Núñez, Rodríguez \& Rubio, 2004): the stimulation to exchange knowledge by answering questions of other users can be weak and professionals may lose their motivation to help others as they can get saturated by a huge amount of questions. The 'market-oriented methodology for discussion forums' by López, Núñez, Rodríguez and Rubio provides a possible solution for these problems. First, once a user has shown that it adds valuable knowledge to the discussion forum, future questions are shown to more experienced professionals and hence it may enhance the probability that the question will be answered. Second, once a certain user has provided significant valuable additions to the discussion forum, the more easy questions will not be shown anymore to that user. This way, the user will be able to save some effort for those questions that really require the skills of the user. A discussion forum based on the 'market-oriented methodology’ might improve knowledge exchange within organizations significantly.

The software applications discussed here show which possibilities are offered to enable intelligent knowledge exchange in a virtual workplace. However, the more fundamental concepts which play a part in both knowledge exchange with or without computer-based support need to be understood. Therefore, possible contexts of knowledge exchange are studied in the following section.

\section{Knowledge Exchange in Context}

Knowledge exchange will take place in specific contexts. If we expect a virtual workplace to support knowledge exchange, we need to understand these contexts better. The context in which knowledge exchange can take place is regarded from a physical, social and digital perspective. The contexts discussed contain possible concepts which are part of the knowledge exchange situations intended, supported by the definition from section 2.2. Specific instantiations of the models discussed below are possible when analyzing knowledge exchange situations in practice. 


\subsection{Using Object Role Modelling (ORM) to Model the Knowledge Exchange Contexts}

We have chosen to model possible knowledge exchange contexts by means of the modelling language 'Object Role Modelling' or 'ORM' (Halpin, 2001; Hofstede, 1993). An important role of the conceptual models depicted in sections 3.2, 3.3 and 3.4 is to provide a common understanding of the Universe of Discourse involved. A Universe of Discourse covers informational aspects of the contexts, while the technical computerized aspects are left out of scope. Thus, the conceptual models introduced in this section cover possible informational aspects when the contexts of knowledge exchange are concerned. It is not our intention to provide a complete representation of knowledge exchange in all its possible contexts (it is assumed that this is not a realistic goal), but to provide more insight in the proposed contexts instead. For clarity, the worker has been positioned within all three contexts.

\subsection{Knowledge Exchange in its Physical Context}

Physical context refers to context about physical properties of knowledge exchange, as can be depicted in figure 1 . At first, a worker requires a communication device to initiate knowledge exchange. A communication device in a physical context can consist of the human vocal cords which can generate verbal signals, but also the head (including eyes and ears) or limbs function as a communication device to communicate non-verbal signals. To exchange knowledge by using a communication device, a transportation medium is required. In case of non-electronic knowledge exchange the air (for transporting vocal sounds) functions as the communication medium. In case of electronic knowledge exchange the hardware interface serves as the communication medium, at least from a physical point of view. The knowledge resources such as a hardware device or a human brain are part of a physical context of knowledge exchange. 


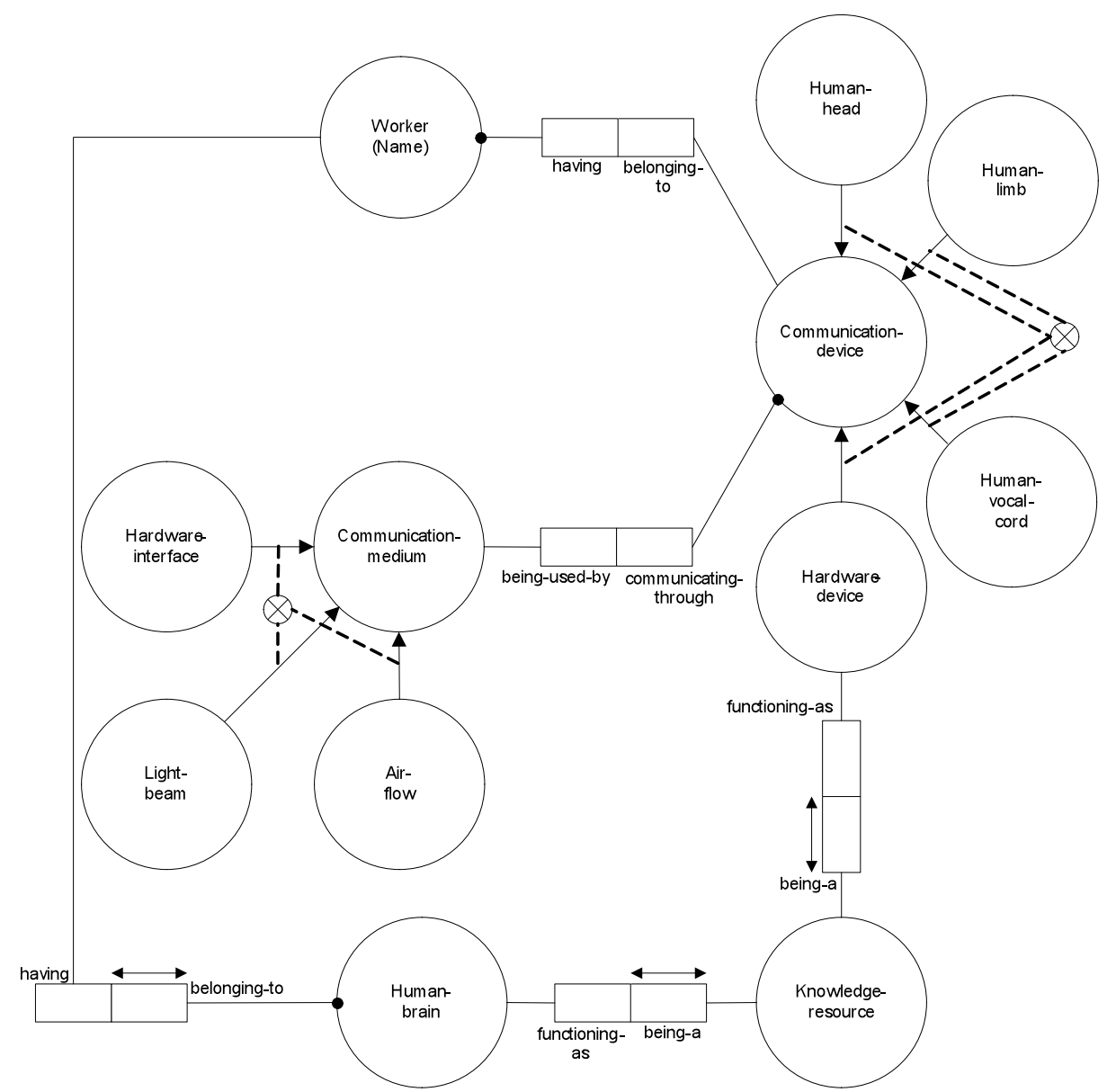

Figure 1: ORM Model of Knowledge Exchange in its Physical Context

Now that a possible physical context has been modelled in figure 1, it is possible to articulate about the so-called deep structure sentences of the ORM-model so that this context can be better understood in practice. Deep structure sentences can be interpreted uniquely if each valid combination Object-Name Role-Name Object-Name has a unique interpretation in the information structure. This is called the Role Identification Rule or also referred to as a (linear) path-expression (Wintraecken, 1990). Complex operations on such sentences may reveal parts of the information structure population one is interested in. Suppose that we are interested in the workers who communicate at least with worker 'Gates' through the air using their voice. The following operation is necessary to retrieve the desired results: Worker having Human-vocal-cord communicating-through Air-flow THAT INCLUDES ALL Air-flow being-used-by Human-vocal-cord belonging to Worker: "Gates". The results of this query may be interesting for analyzing the physical communication lines in an organization. By executing a collection of operations certain knowledge about a physical context of knowledge exchange can be gained, dependent of 
how the ORM-model is populated. This Role Identification Rule exercise at least reveals the following aspects in a physical context of knowledge exchange:

1. Specific physical communication media exist which are used as an interface between workers and physical communication devices.

2. Physical knowledge resources are human brains and hardware devices, which are part of workers respectively used by workers.

\subsection{Knowledge Exchange in its Social Context}

Figure 2 depicts knowledge exchange and the possible objects which play a role in the social context of knowledge exchange. Social context is based on membership in communities and focuses on the relationships of a worker with others, i.e. the social network of a worker (Klein \& Giese, 2005). A social situation consists of individual workers on the one hand and social relationships (who communicates with who) on the other hand. The social network as depicted in figure 2 consists of the social relationships between groups of workers along with the individual workers involved. In a social context, non-linguistic social signals and linguistic social signals play a role in the knowledge exchange process. Non-linguistic social signals consist of body language, facial expression and tone of voice (Pentland, 2004). Linguistic social signals have two perspectives: generative linguistics, also known as Chomskian linguistics and functional linguistics. Research on generative linguistics focuses on the structure of language forms as an isolated topic and functional linguistics aims at describing structural properties of language (both form and meaning) in relation to their function in communication (Hoppenbrouwers, 2003).

Social products are created, institutionalized and made into tradition, into mainstream mental models, by workers in the societies in which they emerge. Mental models are small-scale psychological representations of real, hypothetical or imaginary situations (Craik, 1943). Another aspect in a possible social context of knowledge exchange concerns the different cultural or social backgrounds workers might have, which influences the knowledge a worker possesses. 


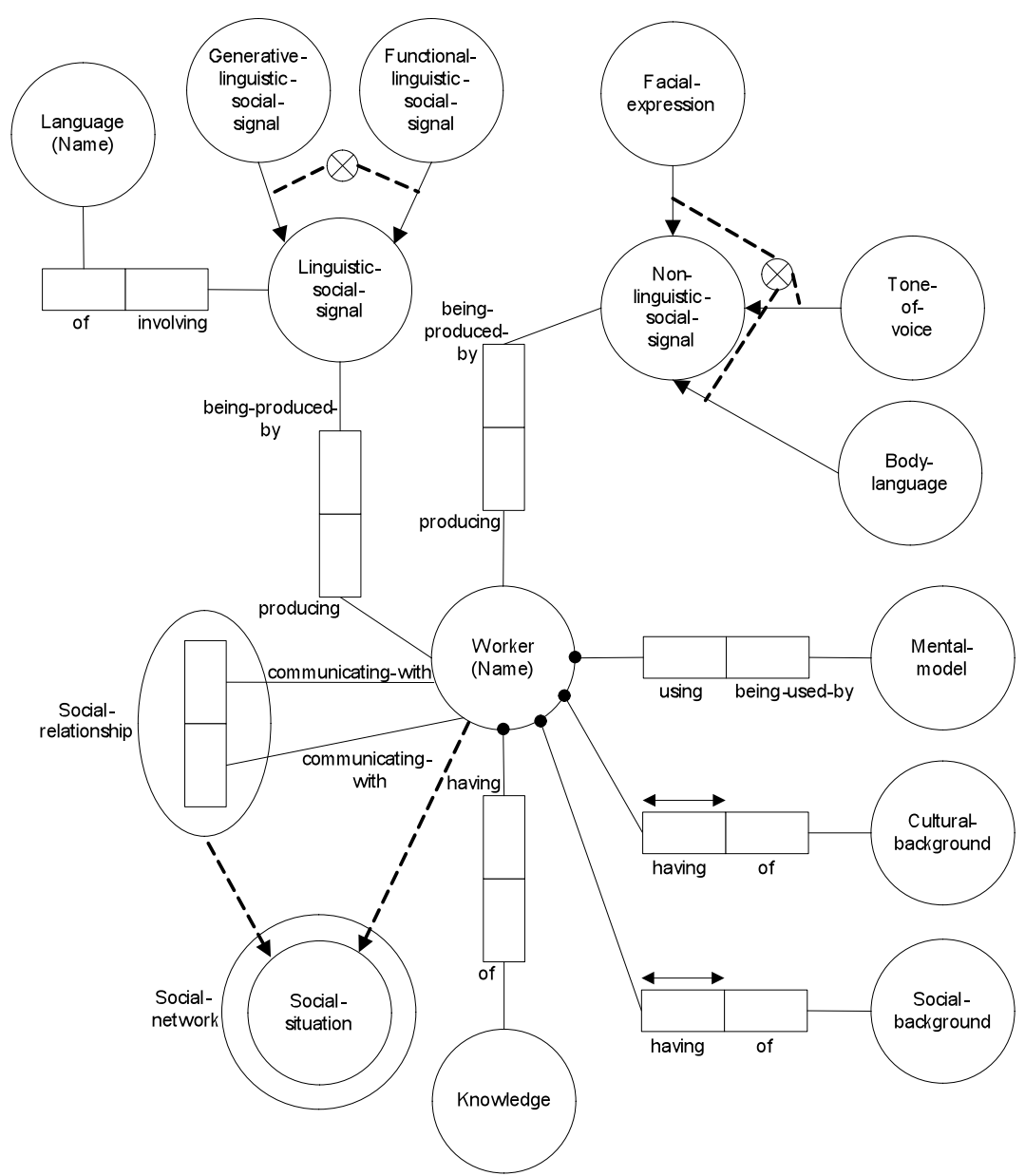

Figure 2: ORM Model of Knowledge Exchange in its Social Context

An interesting operation on the information structure of figure 2 is to gather the workers who communicate with workers who have at least the knowledge of worker 'Gates': Worker communicating-with Worker having Knowledge THAT INCLUDES ALL Knowledge of Worker: "Gates". This is a desirable situation if a certain worker wishes to acquire knowledge which can be provided by at least the worker 'Gates'. Suppose that a virtual workplace interprets the results of the latter query, it can assist the worker in finding the right person to exchange knowledge with. To provide the worker discussed here with appropriate knowledge, possible workers who possess interesting knowledge must have a knowledge profile which equals the knowledge profile of worker 'Gates'. So in terms of the function $N(S, x)$ as introduced in section 2.2, the knowledge profile $G$ of worker 'Gates' must be a subset of a certain knowledge profile $S$, or formally: $G \subseteq S$. The set $G$ can be interpreted as the personal knowledge of the worker 'Gates' (sometimes also called a user profile) during a knowledge exchange session. The set $G$ of already 
presented knowledge then acts as a mini-profile of worker 'Gates' (Weide \& Bommel, 2006). Now several aspects of the proposed social context of knowledge exchange can be determined:

1. A worker possesses specific social properties (knowledge, mental models, a cultural background and a social background).

2. A worker communicates with other workers by producing (non-)linguistic social signals and a social network is formed.

\subsection{Knowledge Exchange in its Digital Context}

Figure 3 shows a possible digital context of knowledge exchange, in which software agents play an important role. A software agent (or agent for short) interacts with other agents or with other workers through software interfaces. When an agent interacts with another agent, an agent relationship is formed. A collection of agents who interact with each other is therefore denoted as an 'agency'. A software agent can make use of a data store for retrieval and storage functions. A data store and a software agent can also function as a knowledge resource if they contain knowledge at a certain moment in time.

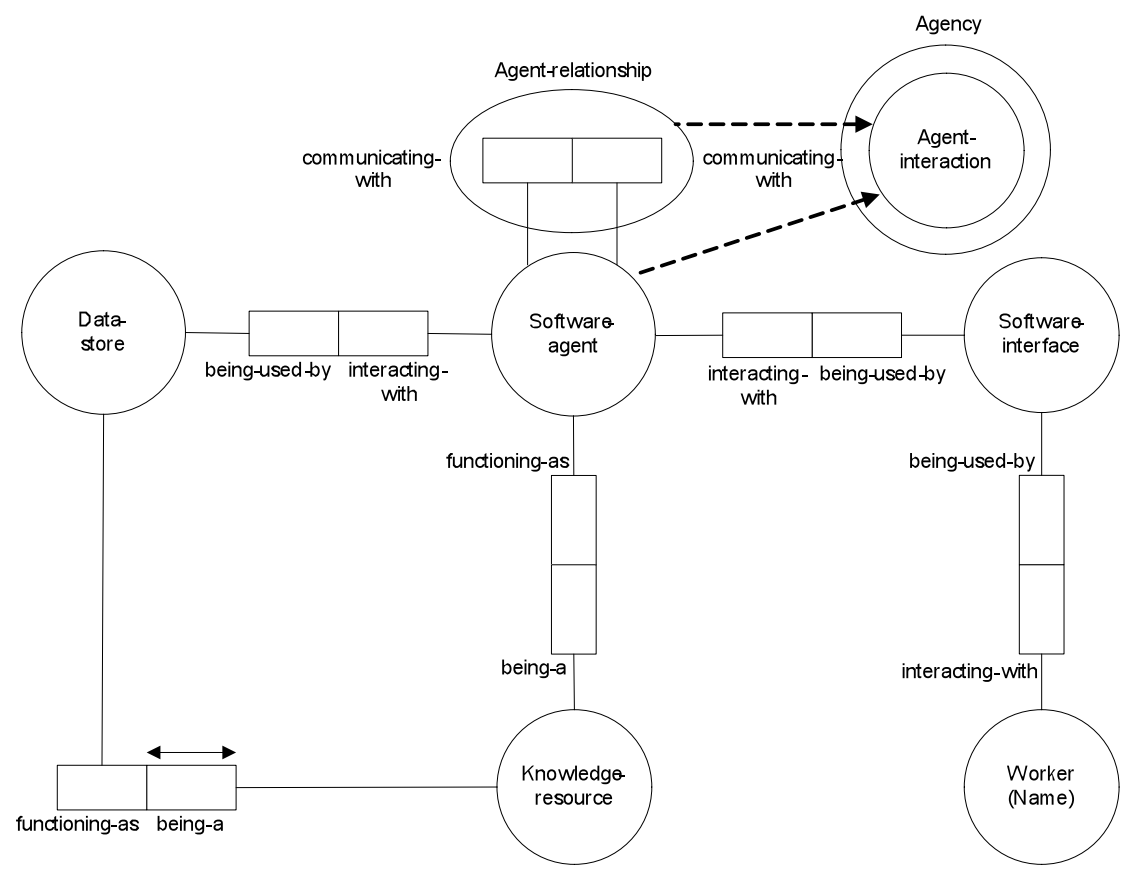

Figure 3: ORM Model of Knowledge Exchange in its Digital Context

An interesting operation on the information structure of figure 3 is expressed by the following sentence: Worker interacting-with Software-interface MATCHING ALL Softwareinterface being-used-by Software-agent: "Agent $\mathbf{n}$ ”. This operation leads to the workers 
who interact with exactly the same software interfaces as 'agent n'. Thus, the query returns the workers who communicate with 'agent n', which might be interesting if one wishes to know with whom 'agent n' exchanges knowledge.

\section{A Fundamental Model of Knowledge Exchange}

After discussing possible contexts in which knowledge exchange takes place, a fundamental model for knowledge exchange will be introduced in this section. This fundamental model consists of three parts: basic knowledge exchange, double party knowledge exchange and an overall framework of knowledge exchange.

\subsection{Basic Knowledge Exchange}

Figure 4 shows how knowledge is exchanged between a worker pair $w_{1}, w_{2} \in W$ using an intervening knowledge set $K$. In section 2.2 we have mentioned that the knowledge input and output consumed respectively generated by a worker during the process of knowledge exchange can be depicted as $i, o: T \rightarrow(W \rightarrow \wp(K))$. In figure 4 , the worker state has been omitted because we only want to focus on knowledge input and output of workers in this basic model. For example, the function $o\left(w_{2}\right)$ depicts the output (in terms of knowledge) of worker $w_{2}$.

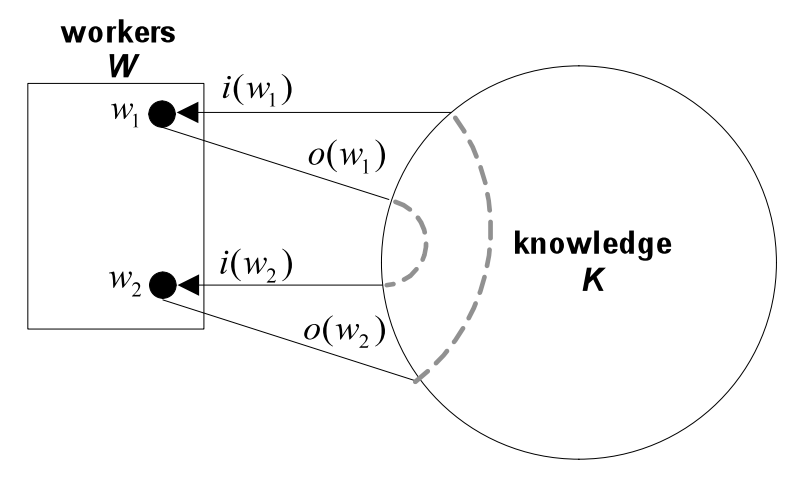

Figure 4: Elementary Forms of Knowledge Exchange

A worker's received respectively broadcasted knowledge items may overlap, which happens if knowledge is exchanged between a worker pair $w_{1}$ and $w_{2}$. Concerning the model of figure 4 , knowledge overlap may occur on four different occasions:

1. $i\left(w_{1}\right) \cap i\left(w_{2}\right) \neq \varnothing$

2. $i\left(w_{1}\right) \cap o\left(w_{2}\right) \neq \varnothing$

3. $o\left(w_{1}\right) \cap i\left(w_{2}\right) \neq \varnothing$

4. $o\left(w_{1}\right) \cap o\left(w_{2}\right) \neq \varnothing$ 
However, the intersection of knowledge items which a worker $w$ exchanges only with himself (such that no new knowledge is gained) is considered as an empty set. Formally, this can be denoted as:

$$
\forall_{w \in W}[i(w) \cap o(w)=\varnothing]
$$

These intersections can be left out of consideration.

\subsection{Double Party Knowledge Exchange via Agents}

The above example involved the knowledge exchange between a worker pair. However, it is less trivial to introduce an additional set of agents which also interacts with a knowledge set $K$. To understand how many forms of knowledge exchange are possible if a virtual workplace is used, consider $A$ as a virtual workplace consisting of agents, where an agent $a \in A$ and $A \neq W$. The introduction of a virtual workplace creates a double party model of knowledge exchange, instead of a single party model as was the case with a worker pair interacting with a knowledge set. Apart from the four elementary forms of knowledge exchange as depicted in section 4.1, there are four additional forms of knowledge exchange between a worker $w \in W$ and an agent $a \in A$ when using a knowledge set $K$ :

1. $i(w) \cap i(a) \neq \varnothing$

2. $i(w) \cap o(a) \neq \varnothing$

3. $o(w) \cap i(a) \neq \varnothing$

4. $o(w) \cap o(a) \neq \varnothing$

\subsection{An Overall Framework of Knowledge Exchange}

In practice, a worker shall deliver knowledge input and output, but agents which are part of the virtual workplace of a worker shall also deliver knowledge input and output. Besides the worker and its virtual workplace, the external environment will also provide input and output. Figure 5 represents an abstract model of knowledge exchange in a more practical situation when compared to the more elementary model of figure 4 . In the remainder of this chapter, the focus is on knowledge exchange in which a worker and a virtual workplace are involved. Possible physical, social and digital contexts such as those mentioned in section 3 involve objects which are part of the external environment as shown in figure 5. 


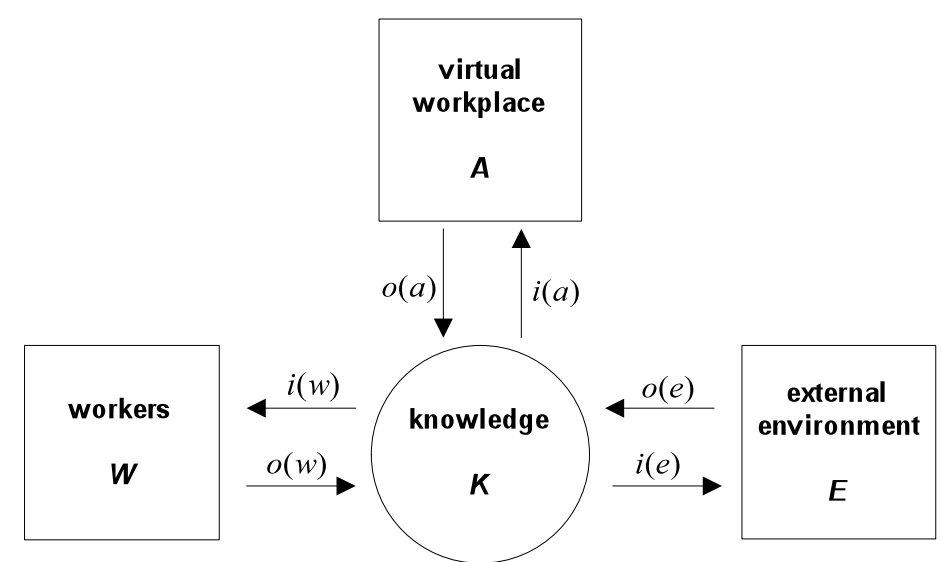

Figure 5: Overall Framework of Knowledge Exchange

In this model $w \in(W), a \in(A)$ and $e \in(E)$. As a result, figure 5 represents a model of knowledge exchange involving three parties.

\section{Enabling Intelligent Knowledge Exchange}

In sections 4.1 and 4.2 we have discussed overlap of knowledge items in the process of knowledge exchange. In this section we will elaborate on that concept and several models for enabling intelligent knowledge exchange are introduced. In this section input and output relatedness, and 1-, 2- and $k$-support relatedness between knowledge items in the process of knowledge exchange are discussed. The models introduced in this section are illustrated with cases from the medical domain.

\subsection{Input and Output Relatedness}

Firstly, the input of a worker $w_{1}$ and the input of a worker $w_{2}$ may be input related. This situation is represented by $i\left(w_{1}\right) \cap i\left(w_{2}\right) \neq \varnothing$. To actually measure the similarities between received knowledge items of workers, the fuzzy logic approach of Jaccard's similarity coefficient can be introduced (Weide \& Bommel, 2006). This coefficient normalizes intersection $i\left(w_{1}\right) \cap i\left(w_{2}\right) \neq \varnothing$ with the corresponding union in case both $i\left(w_{1}\right)$ and $i\left(w_{2}\right)$ are non-empty:

$$
\operatorname{Jacc}\left(i\left(w_{1}\right), i\left(w_{2}\right)\right)=\frac{\left|i\left(w_{1}\right) \cap i\left(w_{2}\right)\right|}{\left|i\left(w_{1}\right) \cup i\left(w_{2}\right)\right|}=\frac{\Sigma_{i} \min \left(s_{i}, t_{i}\right)}{\sum_{i} \max \left(s_{i}, t_{i}\right)}
$$

The fuzzy logic Jaccard's similarity coefficient expresses the degree in which knowledge items $s$ in $i\left(w_{1}\right)$ and knowledge items $t$ in $i\left(w_{2}\right)$ are similar on a [0,1] scale. Overlap between output related knowledge items can also be measured equally. If either $i\left(w_{1}\right)$ or 
$i\left(w_{2}\right)$ is empty, we have $\operatorname{Jacc}\left(i\left(w_{1}\right), i\left(w_{2}\right)\right)=0$. Finally, $\operatorname{Jacc}(\varnothing, \varnothing)=1$. Thus, two possible situations of related knowledge items can be discerned during the process of knowledge exchange: Input related knowledge items and output related knowledge items, as is depicted in figure 6 .
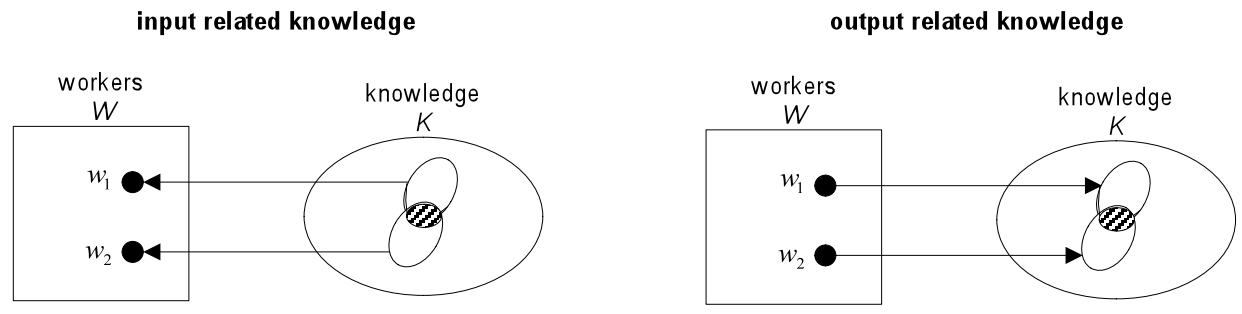

Figure 6: Input and Output Related Knowledge

To illustrate the input and output relatedness as shown in figure 6 , suppose that worker $w_{1}$ and $w_{2}$ are physicians and that worker $w_{1}$ is a radiologist and worker $w_{2}$ is an assistant radiologist. Assume that the radiologist (worker $w_{1}$ ) shows an X-ray of a tuberculosis patient's lungs to the assistant radiologist (worker $w_{2}$ ). The radiologist asks if the assistant can localize and indicate tuberculosis symptoms on the X-ray and thus he generates knowledge output denoted as $o\left(w_{1}\right)$. Worker $w_{2}$ replies with the following output $o\left(w_{2}\right)$ : 'Enlarged lymph nodes present in the bottom right of the X-ray indicating possible tuberculosis'. Now assume that this knowledge was not part of the knowledge profile of the radiologist and that the assistant's answer reduces his need for knowledge concerning the X-ray. In this case, we can speak of 'output related knowledge' between the worker pair, because the assistant produces output which has strong overlap with the output of the radiologist, such that $\operatorname{Jacc}\left(o\left(w_{1}\right), o\left(w_{2}\right)\right)>0$. A similar example can be given for input relatedness, which is considered trivial.

If virtual workplaces keep track of the input and output relatedness between workers, heuristic patterns of overlap between knowledge profiles of workers can be formed over time. This insight in the knowledge profiles of workers can eventually improve knowledge exchange between them.

\subsection{Support Relatedness}

Besides input and output relatedness between knowledge items, 1 -support relatedness between knowledge items can now be introduced. In a 1 -support related situation, a virtual workplace supports a worker by receiving input from the worker and then uses that input to deliver relevant support for the worker. This situation is depicted in figure 7. 
Worker states and agent states are also introduced at this point to keep track of a worker's input and output and an agent's input and output.

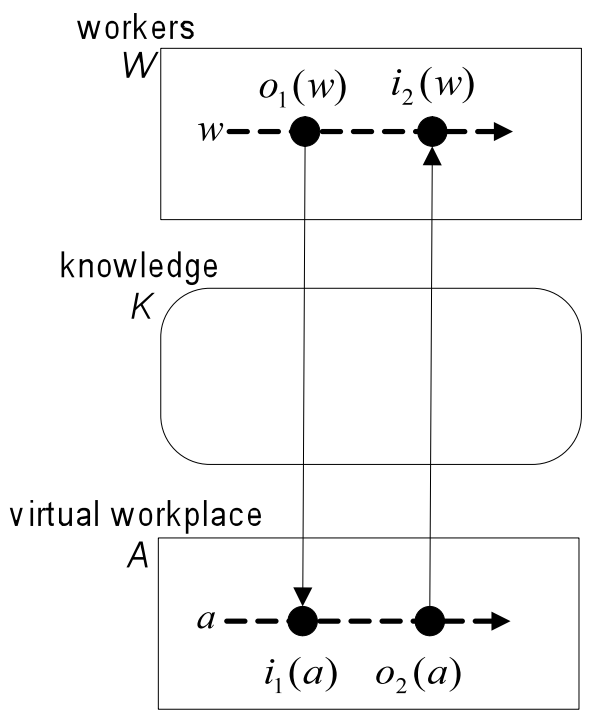

Figure 7: 1-Support Relatedness

The 1 -support situation includes the functions $o_{1}(w) \cap i_{1}(a) \neq \varnothing$ and $o_{2}(a) \cap i_{2}(w) \neq \varnothing$. Considering the function $N: \wp(K) \times K \mapsto[0,1]$ from section 2.2, the initial need for support at the start of a 1 -support situation by a worker is denoted as $N(\varnothing, i(w))$, where $i(w)$ is the input a worker receives from the virtual workplace. As shown in figure 7 , $i_{2}(w)$ is the input which worker $w$ receives as a consequence of output $o_{2}(a)$ in terms of knowledge items. This input should contain additional knowledge compared to the knowledge that a worker had at the start of a 1 -support situation, which is expressed by the following function:

$$
X \subseteq Y \Rightarrow N\left(X, i_{2}(w)\right) \geq N\left(Y, i_{2}(w)\right)
$$

Here, $X$ is the knowledge profile of worker $w$ before receiving support from the virtual workplace and $Y$ is the knowledge profile of worker $w$ after receiving support from the virtual workplace. To illustrate a 1 -support situation in the medical domain, suppose that worker $w$ is still the radiologist. At worker state one, the radiologist would like to know which people within his social network have knowledge about 'tuberculosis symptoms on an X-ray of human lungs', which is expressed by $o_{1}(w)$. The request for knowledge is interpreted by the radiologist's virtual workplace which is denoted as $i_{1}(a)$. Based on a 
match between the input $i_{1}(a)$ and the knowledge profiles which the radiologist's virtual workplace possesses, a selection of profiles are sent (depicted as $o_{2}(a)$ ) and eventually absorbed by the radiologist (depicted as $i_{2}(w)$ ).

If $N\left(Y, i_{2}(w)\right)>0$, then a 2-support situation might be desirable. A 2-support situation, as depicted in figure 8 , complements a 1 -support situation with additional functions $o_{3}(w) \cap i_{3}(a) \neq \varnothing$ and $o_{4}(a) \cap i_{4}(w) \neq \varnothing$.

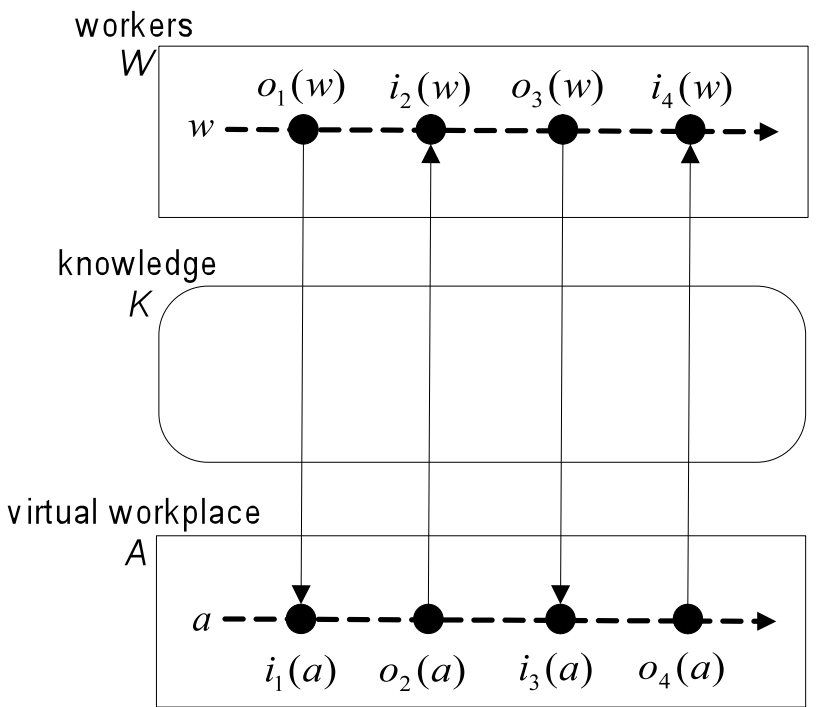

Figure 8: 2-Support Relatedness

Assume the set $Z$ depicts the knowledge which a worker possesses after a 2-support situation has occurred. To illustrate a 2 -support situation in the case of the radiologist mentioned in the 1 -support situation, assume that the selection of profiles which were sent to the radiologist earlier do not satisfy his need for knowledge. Therefore, he would like to retrieve an electronic handbook on the tuberculosis topic and broadcasts this request which is expressed by $o_{3}(w)$. The virtual workplace utilizes an agent to scan all available knowledge resources (including the internet of course) so that the best suitable handbook on the requested topic can be retrieved. Once an electronic handbook has been retrieved, it is broadcasted to the radiologist (depicted by $o_{4}(a)$ ) who receives it (depicted by $i_{4}(w)$ ). Eventually, this ends the 2-support situation if the radiologist is satisfied with the result.

If $N\left(Z, i_{4}(w)\right)>0$, then a $k$-support situation might be desirable. A $k$-support situation simply continues the cycle of providing support to a worker after a 2 -support situation has not resolved certain need for knowledge of a worker. In terms of support relatedness this can be depicted as: 
1. $o_{5}(w) \cap i_{5}(a) \neq \varnothing$

2. $o_{6}(a) \cap i_{6}(w) \neq \varnothing$

3. ...

If a worker has no more need for knowledge, then the possible 1-, 2- and $k$-support situations end.

\section{Future Research}

Verification and validation of the models (which also expands current theory) for intelligent knowledge exchange discussed so far is one of the main challenges of future research. At this stage in the research, a case within the medical domain is sketched to illustrate how a virtual workplace can improve knowledge exchange between physicians based on the theoretical models. The models mentioned in sections 4 and 5 are focused on understanding and enabling intelligent knowledge exchange between workers by means of a virtual workplace. There are no models discussed which propose how a virtual workplace can be implemented or how a virtual workplace should be implemented specifically for a certain community of workers. Further research is needed to clarify those issues.

We have chosen to study a community of physicians in order to verify and also validate the models based on their specific needs for better knowledge exchange. Other community studies are planned in the future to find similarities in verifying and validating the models within different communities of workers. Analysis of those similarities may yield reusable parts of the theoretical models which can be used to improve knowledge exchange within a community of workers in general. A collection of those reusable parts may result in a more abstract model for enabling intelligent knowledge exchange for workers in general. Deploying virtual workplaces is then of course proposed as a solution for improving knowledge exchange. The actual development of a possible prototype of an agent-based virtual workplace which enables intelligent knowledge exchange for physicians can be part of the research in the future.

\section{Conclusion}

An approach to enable intelligent knowledge exchange between workers by means of an agent-based virtual workplace has been elaborated. The approach has been illustrated throughout the chapter by a case from the medical domain. Contemporary physicians witness significant change in the nature of health care delivery and the necessity for them 
to process and disseminate knowledge only increases. After reflecting on the concept of knowledge, a fundamental view on knowledge exchange is elaborated.

Our view is aimed at decreasing a worker's need for knowledge as much as possible and improving the flow of knowledge between workers. It is made clear how the proposed view on knowledge exchange materializes in organizational knowledge transformation processes and organizational knowledge lifecycles. To understand what comprises knowledge exchange in general, insight in possible contexts of knowledge exchange is acquired by introducing several Object Role Modelling (ORM) models. Furthermore, additional theory is introduced to provide a foundation for the support a virtual workplace can deliver for a worker when exchanging knowledge. A practical case shows how a virtual workplace can provide support for a radiologist's knowledge exchange problems in practice. In this case, the radiologist requires knowledge about tuberculosis symptoms.

Further research is necessary to verify and validate the theoretical models in several practical domains. The verification and validation of the theory causes possibilities for improvements and new additions to existing research results.

\section{References}

Barwise, J. (1989). The situation in logic (CSLI Lecture Notes). Stanford, CA: Stanford University, CSLI.

Berliant, M., Reed, R.R., \& Wang, P. (2006). Knowledge exchange, matching, and agglomeration. Journal of Urban Economics, 60(1), 69-95.

Cowan, R., Jonard, N., \& Özman, M. (2004). Knowledge dynamics in a network industry. Technological Forecasting \& Social Change, 71(5), 469-484.

Craik, K. (1943). The nature of explanation. Cambridge, MA: Cambridge University Press.

Dienes, Z., \& Perner, J. (1999). A theory of implicit and explicit knowledge. Behavorial and Brain Sciences, 22(5), 735-808.

Frank, J.R. (Ed). (2005). The CanMEDS 2005 physician competency framework. Better standards. Better physicians. Better care. Ottawa: The Royal College of Physicians and Surgeons of Canada.

Groth, K. (2004). A technological framework supporting knowledge exchange in organizations. In NordiCHI '04: Proceedings of the third Nordic conference on humancomputer interaction (pp. 381-384). New York, NY: ACM Press. 
Halpin, T. (2001). Information modeling and relational databases, from conceptual analysis to logical design. California, CA: Morgan Kaufmann.

Hofstede, A.H.M. ter (1993). Information modelling in data intensive domains. $\mathrm{PhD}$ Thesis. Radboud University, Nijmegen, The Netherlands, EU.

Holsapple, C.W., \& Singh, M. (2001). The knowledge chain model: Activities for competitiveness. Expert Systems with Applications, 20(1), 77-98.

Hoppenbrouwers, S.J.B.A. (2003). Freezing language: Conceptualisation processes in ICT supported organisations. PhD Thesis. Radboud University, Nijmegen, The Netherlands, EU.

Klein, F. \& Giese, H. (2005). Analysis and design of physical and social contexts in multi-agent systems using UML. In SELMAS '05: Proceedings of the fourth international workshop on software engineering for large-scale multi-agent systems (pp. 1-8). New York, NY: ACM Press.

Koulopoulos, T., Spinello, R., \& Toms, W. (1997). Corporate instinct: Building a knowing enterprise for the $21^{\text {st }}$ century. New York, NY: Van Nostrand Reinhold.

Kuznets, S. (1962). Population change and aggregate output. In R. Easterlin (Ed.), Demographic and economic change in developed countries (pp. 324-340). Princeton, NJ: Princeton University Press.

Li, X., Montazemi, A.R., \& Yuan, Y. (2006). Agent-based buddy finding methodology for knowledge sharing. Information \& Management, 43(3), 283-296.

López, N., Núñez, M., Rodríguez, I., \& and Rubio, F. (2004). Encouraging knowledge exchange in discussion forums by market-oriented mechanisms. In $S A C$ '04: Proceedings of the 2004 ACM symposium on applied computing (pp. 952-956). New York, NY: ACM Press.

Malhotra, A., \& Majchrzak, A. (2005). Virtual workspace technologies. MIT Sloan Management Review, 46(2), 11-14.

Nonaka, I., \& Takeuchi, H. (1995). The knowledge-creating company: How Japanese companies create the dynamics of innovation. Oxford, UK: Oxford University Press.

Pentland, A.S. (2004). Social dynamics: Signals and behaviour. In ICDL '04:

Proceedings of the 2004 international conference on developmental learning (pp. 263267). New York, NY: IEEE Press. 
Pinku, G., \& Tzelgov, J. (in press). Consciousness of the self (COS) and explicit knowledge. Consciousness and Cognition.

Polanyi, M. (1966). The tacit dimension. London, UK: Routledge and Kegan Paul.

Schaffers, H., Brodt, T., Pallot, M., \& Prinz, W. (2006). The future workspace: Mobile and collaborative working perspectives. Enschede, The Netherlands: Telematica Instituut.

Siemieniuch, C.E., \& Sinclair, M.A. (1999). Organizational aspects of knowledge lifecycle management in manufacturing. International Journal of Human-Computer Studies, 51(3), 517-547.

Strambach, S. (2001). Innovation processes and the role of knowledge-intensive business services. In K. Koschatzky, M. Kulicke \& A. Zenker (Eds.), Innovation networks concepts and challenges in the European perspective (pp. 53-68). Heidelberg, Germany: Physica-Verlag.

Weide, Th. P. van der, \& Bommel, P. van (2006). Measuring the incremental information value of documents. Information Sciences, 176(2), 91-119.

Wintraecken, J.J.V.R. (1990). The NIAM information analysis method: Theory and practice. Deventer, The Netherlands: Kluwer.

Wooldridge, M., \& Jennings, N. (1995). Intelligent agents: Theory and practice. The Knowledge Engineering Review, 10(2), 115-152.

\section{Glossary of Terms}

Input and output relatedness

Knowledge exchange

Knowledge lifecycle
Input and output relatedness focuses on the existence of overlap between received knowledge and between broadcasted knowledge

Knowledge exchange involves the broadcasting of knowledge items between workers, or between a worker and a software agent and vice versa, with as specific goal to reduce the need for knowledge of a worker

A knowledge lifecycle provides insight in organizational knowledge generation, the propagation of knowledge across the organization, and subsequently the retirement of knowledge 
Knowledge transformation process A knowledge transformation process causes the properties of (a) knowledge item(s) to change and as a result the knowledge item(s) can be classified differently due to the modified properties

Object Role Modelling (ORM) ORM is an information modelling language which has a well-defined formal semantics and sufficient expressive power to describe the Universe of Discourse

Software agent A software agent is an encapsulated computer system that is situated in some environment and that is capable of flexible, autonomous action in that environment

Support relatedness

Support relatedness comprises agent-based theory with as goal to decrease a worker's need for knowledge and to improve the flow of knowledge between workers 